\title{
openheart Hospital meal intake in acute heart failure patients and its association with long-term outcomes
}

\author{
Taizo Yoshida (D) , ${ }^{1}$ Satoshi Shoji, ${ }^{1}$ Yasuyuki Shiraishi, ${ }^{1}$ Masataka Kawana, ${ }^{2}$ \\ Takashi Kohno, ${ }^{3}$ Kenji Inoue, ${ }^{4}$ Keiichi Fukuda, ${ }^{1}$ Paul A Heidenreich, ${ }^{2}$ \\ Shun Kohsaka ${ }^{1}$
}

\begin{abstract}
- Additional material is published online only. To view please visit the journal online (http://dx.doi.org/10.1136/ openhrt-2020-001248).
\end{abstract}

To cite: Yoshida T, Shoji S, Shiraishi Y, et al. Hospital meal intake in acute heart failure patients and its association with long-term outcomes. Open Heart 2020;7:e001248. doi:10.1136/ openhrt-2020-001248

Received 17 January 2020 Revised 12 March 2020 Accepted 23 March 2020
A) Check for updates

(c) Author(s) (or their employer(s)) 2020. Re-use permitted under CC BY-NC. No commercial re-use. See rights and permissions. Published by BMJ.

${ }^{1}$ Department of Cardiology, Keio University School of Medicine, Tokyo, Japan

${ }^{2}$ Department of Medicine, Division of Cardiovascular Medicine, Stanford University, Stanford, California, USA ${ }^{3}$ Department of Cardiovascular Medicine, Kyorin University School of Medicine, Tokyo, Japan

${ }^{4}$ Department of Cardiology, Juntendo University Nerima Hospital, Tokyo, Japan

Correspondence to Dr Shun Kohsaka; sk@keio.jp

\section{ABSTRACT}

Background Risk prediction for hospitalised heart failure (HF, HHF) patients remains suboptimal. We aimed to determine the prognostic value of hospital food intake (FI) immediately before discharge among HHF patients. Method We analysed the data of $255 \mathrm{HHF}$ patients extracted from the records of a single university hospital. The FI percentage of the three meals the day before hospital discharge was averaged. Patients were stratified into adequate $\mathrm{FI}$ (100\% consumption) and inadequate $\mathrm{Fl}$ (less than $100 \%$ consumption) groups. The primary outcome was the composite of all-cause mortality and/or $\mathrm{HF}$ readmission within 1 year.

Results Only $49.3 \%$ of HHF patients consumed $100 \%$ of their meals. Patients with inadequate Fl were older; predominantly women; and had a lower body mass index, higher brain natriuretic peptide levels and Clinical Frailty Scale scores at discharge than those with adequate $\mathrm{Fl}$. Inadequate FI was significantly associated with adverse outcomes after adjustments (HR 2.00; 95\% $\mathrm{Cl} 1.09$ to $3.67 ; p=0.026)$. The effect of interaction by ejection fraction (EF) was highly significant: HF with preserved EF $(\geq 40 \%$ ) was significantly associated with inadequate $\mathrm{FI}$ with adverse outcomes (HR 4.95; 95\% Cl 1.71 to 14.36; $\mathrm{p}=0.003)$ but $\mathrm{HF}$ with reduced $\mathrm{EF}(<40 \%)$ was not (HR $0.77 ; 95 \% \mathrm{Cl} 0.31$ to $1.95 ; p=0.590$ ).

Conclusions The hospital Fl assessment might be a simple, useful tool for predicting and stratifying risk for HHF patients.

\section{INTRODUCTION}

Heart failure (HF) is the leading cause of hospitalisation and is associated with poor prognosis and high medical costs. ${ }^{1-3}$ Risk prediction is of paramount importance to tailor the management of patients with HF. Although numerous conventional risk predictors of HF have been established, such as brain natriuretic peptide (BNP), creatinine and troponin, these parameters require blood sampling and reflect isolated organ dysfunction rather than the general condition of the patient. ${ }^{4-8}$

\section{Key questions}

What is already known about this subject?

- Food intake reflects the general condition of hospitalised patients, and decreased food intake is known to be associated with long-term prognosis among hospitalised patients.

What does this study add?

- This is the first study to demonstrate the association between food intake and the long-term prognosis among hospitalised heart failure patients.

How might this impact on clinical practice?

- The hospital food intake assessment might be a simple, useful tool for predicting and stratifying risk for hospitalised heart failure patients.

Among various nontraditional clinical risk predictors, food intake (FI) is a simple, userfriendly assessment parameter that can be determined at the bedside and is considered to reflect the general condition of hospitalised patients. Several epidemiological studies of hospitalised patients demonstrated that decreased FI represents an independent risk factor for hospital mortality. ${ }^{9}$ Recently, Calleja Fernández et al showed a positive correlation between decreased FI and higher 30-day mortality among haematologyoncology patients. ${ }^{10}$ However, to the best of our knowledge, no studies have reported the association between FI and the mid-term prognosis of hospitalised HF (HHF) patients. Therefore, in this study, we aimed to investigate the prognostic association with hospital FI for HHF patients immediately before their discharge.

\section{METHODS}

This study was conducted as a part of the West Tokyo Heart Failure Registry, ${ }^{11}$ a large, prospective, multicentre cohort registry designed to collect data regarding the clinical 


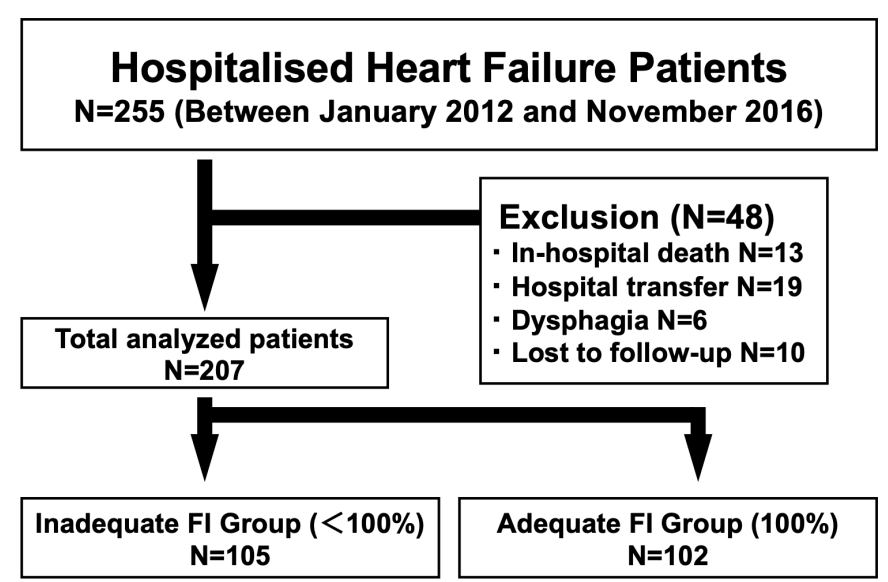

Figure 1 Flow chart of the study design. Adequate food intake $(\mathrm{FI})$ is $100 \%$ consumption of food; inadequate $\mathrm{FI}$ is less than $100 \%$ consumption of food.

backgrounds and outcomes of patients hospitalised for HF. To assess the care and patient outcomes, baseline data and outcomes were collected from individual medical charts by trained clinical research coordinators. Data were entered into an electronic data-capturing system using a robust data query engine and system validations for data quality. Exclusive on-site auditing by the investigators (YS and SK) ensured proper registration by each patient. The objectives and detailed design data are stored on the University Hospital Medical Information Network (UMIN000001171).

\section{Subjects}

The data of 255 consecutive patients who were hospitalised for HF at Keio University Hospital between January 2012 and November 2016 were extracted (figure 1). HHF was defined as rapid-onset HF or a change in HF symptoms requiring urgent therapy and hospitalisation based on the Framingham criteria. ${ }^{1}$ Patients presenting with acute coronary syndrome were not included in this registry. Of the 255 patients, we excluded $13(5.1 \%)$ who died during hospitalisation and, thus, whose mid-term prognosis could not be evaluated; $19(7.5 \%)$ who were transferred to other hospitals for further care and rehabilitation and whose evaluated FI status did not coincide with complete recovery; 6 (2.4\%) with dysphagia whose FI was restricted owing to reasons unrelated to their general status; and $10(3.9 \%)$ who were lost to follow-up. We analysed the remaining 207 patients.

\section{Hospital meal}

Typical hospital meals in Japan consist of a main dish of meat or fish, vegetables and other such food items (figure 2A) and a side dish (bowl of rice). Main meals were plated cold or heated or kept cool in temperaturecontrolled delivery trolleys at the ward level. Salt intake

\section{(A)}

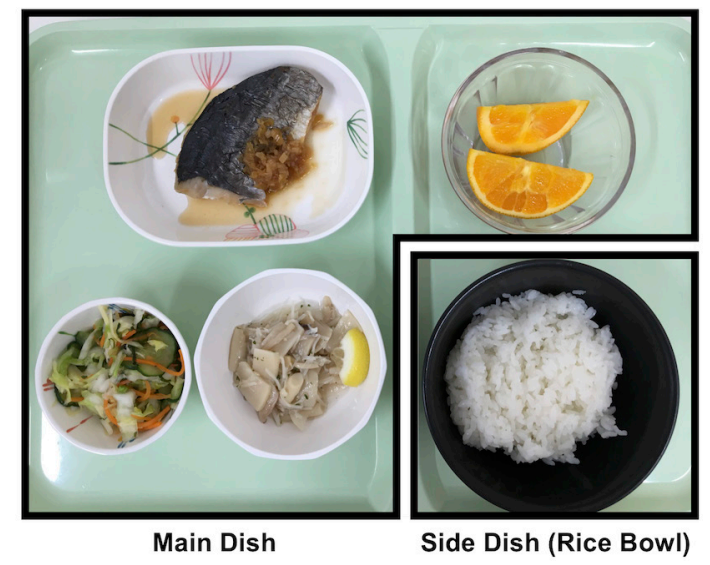

(B)

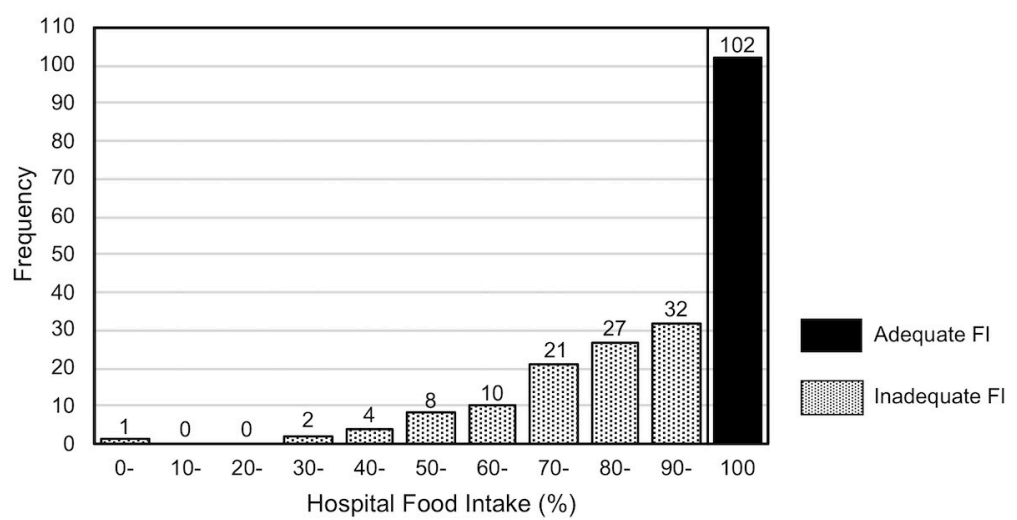

Figure 2 (A) A typical hospital meal in Japan: main dish (fish, vegetables and other food items such as salad and fruit) and side dish (rice bowl). (B) Distribution of food intake percentages $(n=207)$. Adequate $\mathrm{Fl}$ is $100 \%$ consumption of food; inadequate $\mathrm{Fl}$ is less than $100 \%$ consumption of food. 
was limited to $6 \mathrm{~g}$ /day for HHF patients, and protein intake restriction was recommended based on baseline kidney function. Patients were prescribed one of the following therapeutic diets $\geq 24$ hours before the commencement of data collection, thereby allowing sufficient time for the staff to enter the diet prescription: soft, minced and moist, smooth pureed, mildly thick, moderately thick or extremely thick. During the hospitalisation, patients were provided breakfast, lunch and dinner; they were not allowed to bring any food from their home or visit the hospital cafeteria for nutrition education.

\section{Dietary assessment}

Complete dietary provisions and intakes were directly observed, and nurses visually estimated FI percentages (FIP) after each hospital meal (the main dish and rice were estimated separately). This is the standard method in most hospitals in Japan; nurses take the training of standardised visual assessment of FI based on a hospitalwide implemented protocol. ${ }^{12} 13$ This dietary assessment is different from the nutritional assessment performed in the United States, in which a registered dietician routinely performs a 'calorie count' (counting how many calories the patients consumed). ${ }^{14}$ The consumption of each dietary item was recorded as a fraction of the whole portion on a 5-point scale with some flexibility, which has been shown to correlate closely with weighed dietary intake. ${ }^{15}$ One researcher (TY) independently collected all observational and chart audit data to eliminate interrater variability. The overall average percentage of the main dish and side dish intake were calculated for each meal, and FIP was calculated as the average percentage of the three meals (breakfast, lunch and dinner) during the day before hospital discharge. If data for the day before the hospital discharge were not available, then the last three FI data before discharge were used.

\section{Primary outcomes}

The primary outcome was defined as the composite outcome of all-cause death or readmission due to worsening HF within 1 year. Decisions regarding the need for readmission due to HF were made according to the directions of the treating physicians and according to standard practice. The exploratory secondary outcome comprised each separate outcome. Because of the exploratory nature of this study, the sample size was not predetermined.

\section{Definition of other variables}

The Clinical Frailty Scale (CFS) is a generally accepted semiquantitative measure of clinical frailty that can be easily measured by non-geriatricians. ${ }^{16}$ CFS scores were determined according to nurse records by a trained medical professional, according to the Canadian Study of Health and Aging grading criteria. CFS results were categorised into three groups as follows: non-frail (CFS score, 1-3), mildly frail (CFS score, 4-6) and severely frail (CFS score, 7-9).
The Seattle Heart Failure Model (SHFM) is a wellvalidated risk score for predicting the 1-year mortality of HF patients; it is calculated for each patient from 20 variables (age, sex, New York Heart Association functional classification, left ventricular ejection fraction $(\mathrm{EF})$, ischaemic aetiology, systolic blood pressure, diuretic agent dose, angiotensin-converting enzyme inhibitor use, angiotensin receptor blocker use, beta-blocker use, antialdosterone antagonist use, allopurinol use, statin use, device therapy (intracardiac defibrillator (ICD) and cardiac resynchronisation therapy with or without ICD), lymphocyte percentage, serum sodium, total cholesterol, haemoglobin and uric acid). ${ }^{17}$ We previously validated the SHFM externally and internationally among Japanese patients with $\mathrm{HF}^{18}$

Chronic kidney disease was defined as either kidney damage or a decreased estimated glomerular filtration rate (eGFR) of $<60 \mathrm{~mL} / \mathrm{min} / 1.73 \mathrm{~m}^{2}$ for at least 3 months. ${ }^{19}$ Plasma BNP levels were measured on admission and at discharge.

\section{Statistical analyses}

Initially, the distribution of the FIP for the entire population was described (figure 2B). The association between FIP and CFS scores was assessed using Spearman's correlation coefficient (online supplementary figure 1). Patients were categorised into two groups: inadequate FIP group (less than $100 \%$ consumed) and adequate FIP group ( $100 \%$ consumed) (table 1). Normality of continuous variables was tested using the Shapiro-Wilk test and/or Q-Q plot visual assessment. Continuous variables with normal distributions were expressed as mean $\pm \mathrm{SD}$. Other variables were expressed as median (25th and 75th percentiles). Differences between groups were tested using Student's t-tests or Mann-Whitney U tests for continuous variables and Pearson $\mathrm{X}^{2}$ tests for categorical variables. Patients with $\mathrm{HF}$ were also classified as those with $\mathrm{HF}$ with preserved $\mathrm{EF}$ (HFpEF, $\mathrm{EF} \geq 40 \%$ ) and $\mathrm{HF}$ with reduced $\mathrm{EF}$ (HFrEF, $\mathrm{EF}<40 \%$ ). Moreover, baseline characteristics of the inadequate FIP group and the adequate FIP group were compared (online supplementary tables 1 and 2). In addition, the event-free rate of the primary outcome was estimated using the KaplanMeier method, and differences were assessed with the log-rank test (figure 3). To estimate the adjusted HRs of the association between FIP and the primary outcome, the Cox proportional hazards analysis was performed after adjusting for SHFM. In addition to the above, we conducted the subanalysis adjusting for SHFM and BNP levels at discharge.

The association of the primary outcome with FI of the main dish and side dish was evaluated (online supplementary figure 2). Associations of FIP with individual components of the primary outcome (all-cause death and HF readmission) were also assessed (online supplementary figure 3A). To evaluate the robustness of our findings, a sensitivity analysis using different FIP cut-off values (90\%) was performed (online supplementary figure 3B). Finally, an adjusted analysis of the subgroups relevant to $\mathrm{HF}$ 
Table 1 Baseline characteristics of the study population

\begin{tabular}{|c|c|c|c|}
\hline & Inadequate FIP $(<100 \%) n=105$ & Adequate FIP $(100 \%) n=102$ & $P$ value \\
\hline Age, years & $77.7 \pm 11.4$ & $69.8 \pm 13.3$ & $<0.001$ \\
\hline Female, \% & $60(57.1)$ & $30(29.4)$ & $<0.001$ \\
\hline $\mathrm{BMI}, \mathrm{kg} / \mathrm{m}^{2}$ & $20.9 \pm 3.5$ & $22.8 \pm 4.1$ & 0.001 \\
\hline \multicolumn{4}{|l|}{ NYHA at discharge } \\
\hline NYHA I, \% & $13(12.4)$ & $18(17.6)$ & 0.288 \\
\hline NYHA II, \% & $72(68.6)$ & $75(73.5)$ & 0.432 \\
\hline NYHA III, \% & $20(19.0)$ & $9(8.8)$ & 0.034 \\
\hline Hypertension, \% & $75(72.8)$ & $71(70.3)$ & 0.690 \\
\hline Dyslipidaemia, \% & $35(34.0)$ & $33(33.7)$ & 0.963 \\
\hline COPD, \% & $9(8.6)$ & $8(8.0)$ & 0.882 \\
\hline Dialysis, \% & $4(3.8)$ & $1(1.0)$ & 0.369 \\
\hline Diabetes mellitus, \% & $33(31.4)$ & $36(35.6)$ & 0.522 \\
\hline Smoking, \% & $16(15.7)$ & $27(27.0)$ & 0.050 \\
\hline Previous HF Admission, \% & $22(21.2)$ & $17(16.8)$ & 0.431 \\
\hline Systolic BP, mm Hg & $112 \pm 18$ & $109 \pm 16$ & 0.279 \\
\hline \multicolumn{4}{|l|}{ Laboratory data at discharge } \\
\hline Creatinine, $\mathrm{mg} / \mathrm{dL}$ & $1.08(0.86-1.58)$ & $1.04(0.91-1.27)$ & 0.390 \\
\hline Haemoglobin, $\mathrm{g} / \mathrm{L}$ & $113(101-129)$ & $129(113-145)$ & $<0.001$ \\
\hline Sodium, $\mathrm{mEq} / \mathrm{L}$ & $140(137-141)$ & $139(138-142)$ & 0.480 \\
\hline Potassium, $\mathrm{mEq} / \mathrm{L}$ & $4.4(4.1-4.7)$ & $4.4(4.2-4.7)$ & 0.369 \\
\hline BUN, mg/dL & $27.4(19.7-37.5)$ & $24.3(20.0-30.5)$ & 0.135 \\
\hline BNP, pg/uL & $306(191-612)$ & $185(81.6-353)$ & $<0.001$ \\
\hline eGFR, $\mathrm{mL} / \mathrm{min} / 1.73 \mathrm{~m}^{2}$ & $42.2(25.9-56.0)$ & $51.0(40.5-61.2)$ & 0.002 \\
\hline Albumin, mg/dL & $3.4(3.2-3.8)$ & $3.7(3.3-3.9)$ & 0.003 \\
\hline$E F, \%$ & $48(33-60)$ & $40(29-57)$ & 0.012 \\
\hline HFpEF (EF $\geq 40 \%), \%$ & $68(64.8)$ & $47(46.1)$ & 0.007 \\
\hline HFrEF (EF < $<0 \%), \%$ & $37(35.2)$ & $55(53.9)$ & 0.007 \\
\hline CFS score $1-3, \%$ & $10(9.5)$ & $21(20.6)$ & 0.026 \\
\hline CFS score 4-6, \% & $90(85.7)$ & $79(77.5)$ & 0.125 \\
\hline CFS score 7-9, \% & $5(4.8)$ & $2(2.0)$ & 0.265 \\
\hline Calcium channel blocker, \% & $40(38.1)$ & $27(26.5)$ & 0.074 \\
\hline ACE inhibitor, $\%$ & $28(26.7)$ & $34(33.3)$ & 0.295 \\
\hline ARB, $\%$ & $36(34.3)$ & $45(44.1)$ & 0.147 \\
\hline Beta blocker, \% & $83(79.0)$ & $84(82.4)$ & 0.547 \\
\hline SHFM-predicted 1-year survival, \% & $91.4(87.4-94.6)$ & $94.4(90.9-96.4)$ & $<0.001$ \\
\hline Length of hospital stay & $13.0(9.0-21.5)$ & $13.0(10.0-20.3)$ & 0.600 \\
\hline
\end{tabular}

Values are $\mathrm{n}(\%)$, mean $\pm \mathrm{SD}$ or median (25th and 75 th percentiles).

ACE, angiotensin-converting enzyme; ARB, angiotensin receptor blocker; BMI, body mass index; BNP, brain natriuretic peptide; BP, blood pressure; BUN, blood urea nitrogen; CFS, Clinical Frailty Scale; COPD, chronic obstructive pulmonary disease; EF, ejection fraction; eGFR, estimated glomerular filtration rate; FIP, food intake percentage; HF, heart failure; HFpEF, heart failure with preserved ejection fraction; HFrEF, heart failure with reduced ejection fraction; NYHA, New York Heart Association; SHFM, Seattle Heart Failure Model.

prognosis was performed; the relevant variables were age (stratified by 75 years), sex, body mass index (BMI; stratified by $23 \mathrm{~kg} / \mathrm{m}^{2}$ ), chronic kidney disease (stratified by $60 \%$ eGFR), EF (cut-off, 40\%), BNP (stratified by the median) and albumin (stratified by the median) (online supplementary figure 4 ). All p values were two sided. Results were considered statistically significant at $p<0.05$. All statistical analyses were performed using SPSS V.24.0 (IBM). 
(A)

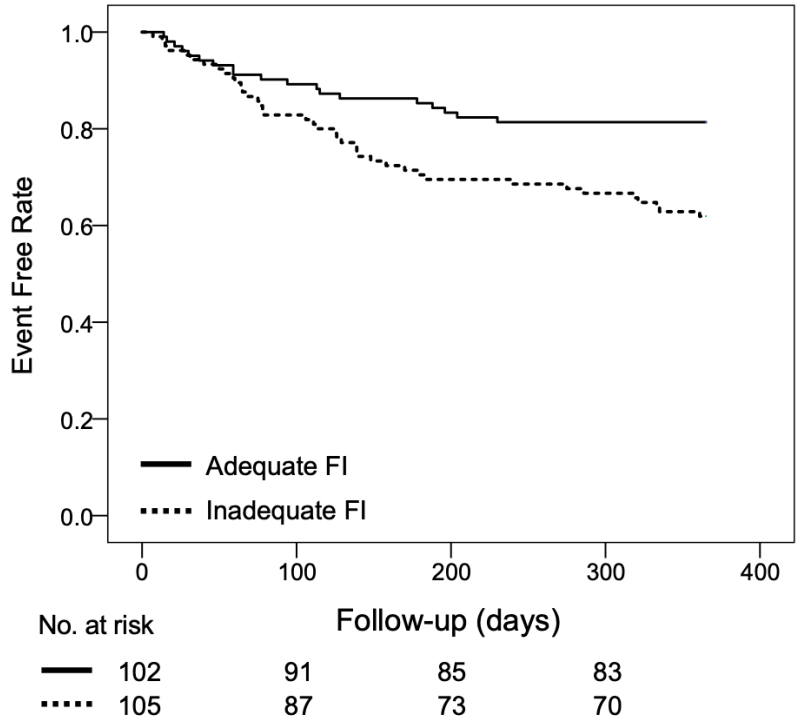

(B)
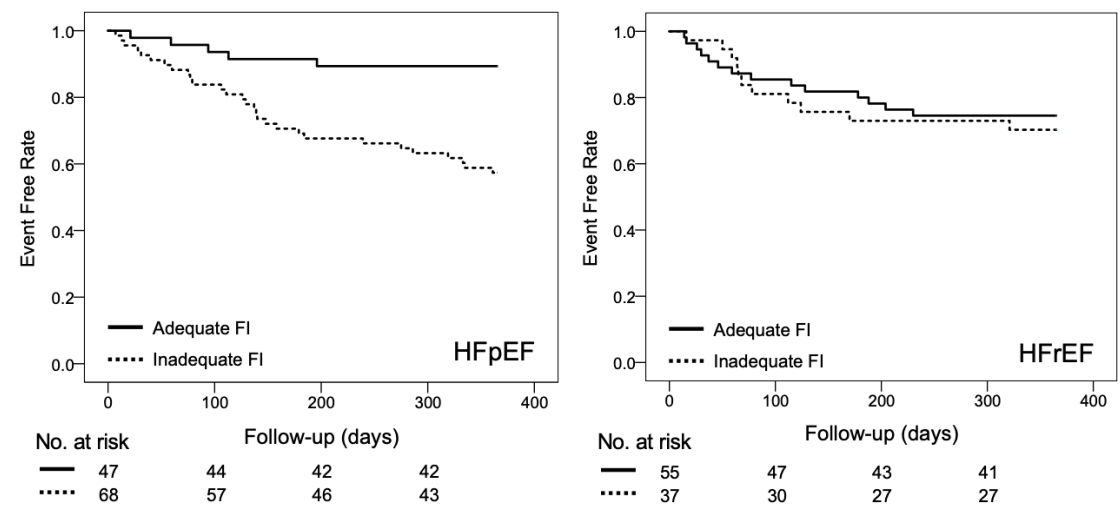

Figure 3 (A) Kaplan-Meier survival curves for the primary outcome (all-cause death or heart failure readmission within 1 year). (B) Kaplan-Meier curves for the primary outcome according to the heart failure phenotypes: HFpEF (EF $\geq 40 \%)$ and heart failure with reduced $\mathrm{EF}(<40 \%)$. Adequate food intake $(\mathrm{FI})$ is $100 \%$ consumption of food; inadequate $\mathrm{FI}$ is less than $100 \%$ consumption of food. EF, ejection fraction; HFpEF, heart failure with preserved EF; HFrEF, heart failure with reduced EF.

\section{RESULTS}

The mean age of the patients was 74 years, and $43 \%$ were women. Table 1 shows the baseline characteristics stratified by FIP. Compared with patients in the adequate FIP group, patients in the inadequate FIP group were older, predominantly female, had lower BMI, had lower haemoglobin levels, had lower eGFR and had higher CFS scores. Moreover, the inadequate FIP group had higher BNP levels at discharge (inadequate FIP: 306 (range, 191-612) $\mathrm{pg} / \mathrm{mL}$; adequate FIP: 185 (range, 81.6-353) pg/mL; $\mathrm{p}<0.001)$ and lower BNP change rates during hospitalisation (inadequate FIP: $49.3 \%$ (range, 29.2\%-64.7\%); adequate FIP: $68.1 \%$ (range, $43.3 \%-83.1 \%$ ); p=0.001) (online supplementary figure 5). Baseline characteristics of HFpEF and HFrEF patients stratified by FIP are shown in online supplementary tables 1 and 2.

Median FIP was 98.3\% (IQR, 81.7\%-100\%) (figure 2B), with 105 patients $(50.7 \%)$ in the inadequate FIP group and 102 patients $(49.3 \%)$ in the adequate FIP group. The association between FIP and CFS is shown in online supplementary figure 1. Spearman's correlation coefficient revealed a significant negative correlation between FIP and CFS $(r=-0.29 ; p<0.001)$.
A total of 59 patients $(28.5 \%)$ attained the primary outcome: 40 were in the inadequate FIP group and 19 were in the adequate FIP group. The Kaplan-Meier curves showed that patients in the inadequate FIP group had a higher incidence of achieving the primary outcome (log-rank test; $p=0.003$ ) than those in the adequate FIP group (figure 3A). The association was preserved after the Cox regression analysis (HR 2.00; 95\% CI 1.09 to $3.67 ; \mathrm{p}=0.026$ ). In the subanalysis adjusted for SHFM and BNP levels at discharge, the result was not statistically significant but directionally similar to our main finding (HR 1.69; 95\% CI 0.82 to 3.45; $\mathrm{p}=0.153$ ). HFpEF (EF $\geq 40 \%$ ) was significantly associated with inadequate FI and the primary outcome (HR 4.95; 95\% CI 1.71 to 14.36 ; $\mathrm{p}=0.003)$, whereas HFrEF $(\mathrm{EF}<40 \%)$ was not associated with FI (HR 0.77; 95\% CI 0.31 to $1.95 ; \mathrm{p}=0.590)$; the test for interaction with $\mathrm{EF}$ was highly significant $(\mathrm{p}=0.030)$ (figure 3B). A sensitivity analysis using a different EF cut-off value $(50 \%)$ demonstrated the robustness of the result; HFpEF ( $\mathrm{EF} \geq 50 \%$ ) was significantly associated with inadequate FI and the primary outcome (HR 5.58; 95\% CI 1.64 to $19.04 ; \mathrm{p}=0.006)$ but $\mathrm{HFrEF}(\mathrm{EF}<50 \%)$ was not (HR $0.95 ; 95 \%$ CI 0.42 to $2.11 ; \mathrm{p}=0.889$ ). 
When analysed individually, inadequate intake of the main dish was significantly associated with adverse outcomes (HR 2.36; $95 \%$ CI 1.31 to 4.25 ; $\mathrm{p}=0.004$ ), whereas inadequate side dish intake showed only a trend for associations (HR $1.60 ; 95 \%$ CI 0.90 to $2.84, \mathrm{p}=0.106$ ) (online supplementary figure 2). There were associations of FIP and individual components of the primary outcome (all-cause death and HF readmission within 1 year; online supplementary figure 3A). In total, 23 patients died during the 1-year follow-up, including 14 patients in the inadequate FIP group and nine patients in the adequate FIP group. Inadequate FIP was not associated with all-cause mortality within 1 year (HR 1.46; $95 \%$ CI 0.60 to $3.57 ; \mathrm{p}=0.409$ ). In contrast, 48 patients were readmitted to the hospital for worsening HF within 1 year, including 32 patients from the inadequate FIP group and 16 patients from the adequate FIP group. Notably, patients from the inadequate FIP group were more likely to be readmitted to the hospital due to worsening HF; however, this was not statistically significant (HR 1.80; 95\% CI 0.91 to $3.55 ; \mathrm{p}=0.093$ ). A sensitivity analysis using a different FI cut-off $(90 \%)$ showed similar results concerning the main result, indicating that inadequate FIP was significantly associated with the primary outcome (HR 1.84; $95 \%$ CI 1.05 to 3.23; $\mathrm{p}=0.033$ ) (online supplementary figure $3 \mathrm{~B}$ ). Finally, subgroup analyses showed that the inadequate FIP group was consistently more likely to have a poor prognosis across various subgroups (online supplementary figure 4).

\section{DISCUSSION}

In this study, we demonstrated the following main findings: only half of the HHF patients were able to consume all three meals on the day before hospital discharge; patients with inadequate FIP had higher BNP levels at discharge and lower BNP improvement rates during hospitalisation; there was a significant correlation between FI and CFS; inadequate FIP was associated with a higher incidence of death and readmission due to $\mathrm{HF}$ within 1 year; the test for the interaction between HFpEF and HFrEF was significant; HFpEF patients with low FI had significantly higher adverse outcomes, but HFrEF patients did not. Therefore, our study provided evidence that FIP might be used as a prognostic indicator for HHF patients, thereby providing a simple and novel risk stratification method for HHF patients.

To the best of our knowledge, no studies have evaluated the association between FIP and the mid-term prognosis for HHF patients. However, several multicentre observational surveys performed in the field of epidemiology have shown that insufficient intake of the provided food was associated with adverse outcomes. Hiesmayr et al demonstrated that decreased FI represented an independent risk factor for hospital mortality of general hospitalised patients. ${ }^{9}$ Tangvik et al identified decreased FI as a predictor of morbidity and mortality within 1 year among general hospitalised patients. ${ }^{20}$ Fernandez et al showed an upward trend between decreased FI and higher 30-day mortality among hematology-oncology patients. ${ }^{10}$
Thibault et al also demonstrated that patients with a poor nutritional status had more healthcare-associated infections than those with normal nutritional status. ${ }^{21}$ Energy deficit during the first week of admission was associated with an increased proportion of infections for patients who were in the intensive care unit. ${ }^{22}$ The results of these studies support the conclusion that inadequate FI is associated with a poor prognosis and are in agreement with our findings for HHF patients.

A significant interaction effect was noted for the HF phenotype (HFpEF vs HFrEF). HFpEF is highly heterogeneous and influenced by a range of cardiac and non-cardiac comorbidities (eg, hypertension, diabetes and atrial fibrillation) typically experienced by elderly patients. These comorbidities lead to systematic microvascular inflammation, which adversely affects the adjacent cardiomyocyte through decreased nitric oxide bioavailability, reduced cyclic guanosine monophosphate availability and altered phosphorylation of titin. ${ }^{23} 24$ Because such systemic microvascular inflammation leads to impaired myocardial energetics and decreased nutritional status, ${ }^{25}$ low FI might be a stronger predictor for HFpEF patients in our study. Additionally, because of the increasing number of HFpEF patients in the contemporary cardiovascular practice and the neutral results of large-scale, randomised controlled trials that tested conventional HF therapies, the results of this study provide crucial evidence that could lead to guidelines that enable better care for HFpEF patients. On the other hand, the inadequate FIP group was not associated with adverse outcomes in HFrEF patients. As shown in online supplementary tables 1 and 2, HFrEF patients were younger and less frail than HFpEF patients in our cohort. Therefore, the inadequate FI, considered as indicative of frailty for HFrEF patients, might not be the case when compared with that for HFpEF patients. Moreover, the previous studies that evaluated the association between FIP and adverse outcomes were performed among relatively severe condition populations, ${ }^{10}{ }^{22}$ suggesting the utility of FIP among HFpEF patients rather than that among HFrEF patients.

Inadequate intake of the main dish showed a significant association with adverse outcomes, whereas inadequate intake of rice was not significantly associated with adverse outcomes. The difference between the main dish and rice may be that rice, a staple food in Japan, is readily consumed by most Japanese, including patients with severe systemic illness. Therefore, analysing the intake of the main dish and rice separately excluded the possible effect of patients' food preferences, thereby strengthening the utility of FI as a potential prognostic indicator.

The association of inadequate FI with a worse mid-term prognosis observed in our study could be explained by several mechanisms. First, inadequate FI might reflect insufficient congestion reduction. Patients with inadequate FIP had higher BNP levels at discharge and lower BNP improvement rates during hospitalisation than patients with adequate FIP (table 1 and online supplementary 
figure 5). This suggested that patients in the inadequate FI group might have had persistent clinical or subclinical congestion. From a physiological viewpoint, patients with persistent congestion have pulmonary oedema, which causes difficulty in breathing during meals and intestinal mucosal oedema related to persistent systemic congestion. Persistent intestinal congestion may lead to reduced secretion of ghrelin, a hormone that enhances growth hormone secretion in the pituitary gland, induces hyperphagia and gastrointestinal motility, and has cardioprotective effects from the intestinal tract, resulting in anorexia. ${ }^{26}{ }^{27}$ Therefore, inadequate FIP may be a manifestation of persistent subclinical congestion in HHF patients. The main cause of rehospitalisation for HF was reported to be attributable to poor adherence to a low-salt diet ${ }^{28}$; therefore, it is difficult to control the appropriate salt intake among patients with HF with persistent congestion and inadequate FI. Further intervention, such as postponement of discharge or implementation of additional medication titration, such as furosemide or tolvaptan, could be considered to improve the prognosis. Inadequate FI is also indicative of frailty, as indicated by our finding of a significant correlation between FI and CFS. Frailty is associated with ageing; it is defined as a decreased physiologic and cognitive function resulting in physical and mental vulnerability to stressors. ${ }^{29}{ }^{30}$ For elderly patients with HF, frailty increases the risk of adverse health events. ${ }^{31}$ Moreover, appropriate early intervention, such as cardiac rehabilitation, aerobic exercise, nutrition education and patient education, reversibly improves the functional capacity of frail HF patients. ${ }^{32}$ Frailty is also an important consideration during the assessment before advanced HF therapies, including ventricular assist device implantation or heart transplantation..$^{33}$ Our results are important not only for the prognostication of HF patients but also for frailty assessments.

Our findings should be interpreted in the context of several limitations and considerations. First, because this was an observational study, some unmeasured or unmeasurable variables might have influenced the outcomes. Nevertheless, the robustness of our results was confirmed through rigorous statistical analyses, namely, the sensitivity and subgroup analyses. Second, because of the small sample size, some subgroup analyses with statistically non-significant results may have been underpowered. Third, this study was conducted at a single university-based hospital, which might have affected the external validity of the results. Nevertheless, the baseline characteristics between our population and large-scale Japanese registries were very similar, ${ }^{2}$ suggesting that our results could be generalised across Japan. Additionally, although hospital meals differ between countries and cultures, FI is universally representative of the systemic status, and various studies of FI have been performed worldwide. $.^{9} 10121520-22$ Fourth, the cause of reduced FIP was not examined in this study. Factors, such as patients' food preference as well as the lack of FI and patients' unwillingness or inability to consume food (eg, due to malignancies), might have affected their FI. Fifth, although the required calorie intake varies with many factors (eg, age, sex, BMI and activities of daily living), we could not examine the individual adequate calorie intake in this study. However, the Harris-Benedict equation was used to estimate basal energy expenditure of individuals adjusted for height, weight, age and sex, and hospital meals were optimised for individual patients. ${ }^{34}$ Sixth, we could not examine the correlation between FI and the data regarding the physical examination and chest X-ray at discharge because these data were not collected in our database. However, as an alternative measure, we have investigated the association of inadequate FIP with BNP levels at discharge and BNP change rates during hospitalisation, as BNP is shown to be better surrogates for congestion. ${ }^{35}{ }^{36}$ Finally, we investigated FIP only on the day before discharge, and changes in FIP during the hospitalisation may have different implications.

In conclusion, inadequate FI by HHF patients is associated with a higher incidence of all-cause mortality or HF readmission within 1 year. Therefore, monitoring FI may be useful for predicting and stratifying risk for HHF patients because it is routinely recorded, simple to perform and economical.

Contributors The author contributions are as follows: Planning and conducting the research: TY, SS, YS and SK. Reporting of the work: TY, SS and YS. Critical revision of the manuscript for important intellectual content: MK, TK, KI, KF and PAH.

Funding This work was supported by a grant from the Japan Agency for Medical Research and Development (201439013C (SK)), Health Labor Sciences Research Grant (14528506; (SK)), a Grant-Aid for Scientific Research (KAKENHI, 18 K08056 (SK)) and a Grant-in-Aid for Young Scientists (JPSS KAKENHI, 18K15860 (YS)).

Competing interests SK reports investigator-initiated grant funding from Bayer and Daiichi Sankyo. The other authors declare no conflict of interest.

Patient consent for publication Not required.

Ethics approval The study protocol was approved by the Keio University Institutional Review Board, and the research was conducted in accordance with the Declaration of Helsinki.

Provenance and peer review Not commissioned; externally peer reviewed. Data availability statement Data are available on reasonable request.

Open access This is an open access article distributed in accordance with the Creative Commons Attribution Non Commercial (CC BY-NC 4.0) license, which permits others to distribute, remix, adapt, build upon this work non-commercially, and license their derivative works on different terms, provided the original work is properly cited, appropriate credit is given, any changes made indicated, and the use is non-commercial. See: http://creativecommons.org/licenses/by-nc/4.0/.

\section{ORCID iD}

Taizo Yoshida http://orcid.org/0000-0003-4965-7495

\section{REFERENCES}

1 Yancy CW, Jessup M, Bozkurt B, et al. 2013 ACCF/AHA guideline for the management of heart failure: Executive summary: a report of the American College of cardiology Foundation/American heart association Task force on practice guidelines. Circulation 2013;128:1810-52.

2 Shiraishi Y, Kohsaka S, Sato N, et al. 9-Year Trend in the Management of Acute Heart Failure in Japan: A Report From the National Consortium of Acute Heart Failure Registries. J Am Heart Assoc 2018;7:e008687.

3 Hollenberg SM, Warner Stevenson L, Ahmad T, et al. 2019 ACC expert consensus decision pathway on risk assessment, management, and clinical trajectory of patients hospitalized 
with heart failure: a report of the American College of cardiology solution set oversight Committee. J Am Coll Cardio 2019;74:1966-2011.

4 de Lemos JA, McGuire DK, Drazner MH. B-Type natriuretic peptide in cardiovascular disease. Lancet 2003;362:316-22.

5 York MK, Gupta DK, Reynolds CF, et al. B-Type Natriuretic Peptide Levels and Mortality in Patients With and Without Heart Failure. J Am Coll Cardiol 2018;71:2079-88.

6 Löffler Al, Cappola TP, Fang J, et al. Effect of renal function on prognosis in chronic heart failure. Am J Cardiol 2015;115:62-8.

7 Nagarajan V, Hernandez AV, Tang WHW. Prognostic value of cardiac troponin in chronic stable heart failure: a systematic review. Heart 2012;98:1778-86.

8 Myhre PL, O'Meara E, Claggett BL, et al. Cardiac troponin I and risk of cardiac events in patients with heart failure and preserved ejection fraction. Circ Heart Fail 2018;11:e005312.

9 Hiesmayr M, Schindler K, Pernicka E, et al. Decreased food intake is a risk factor for mortality in hospitalised patients: the NutritionDay survey 2006. Clin Nutr 2009;28:484-91.

10 Calleja Fernández A, Pintor de la Maza B, Vidal Casariego A, et al. Food intake and nutritional status influence outcomes in hospitalized hematology-oncology patients. Nutr Hosp 2015;31:2598-605.

11 Shiraishi Y, Kohsaka S, Abe T, et al. Validation of the get with the Guideline-Heart failure risk score in Japanese patients and the potential improvement of its discrimination ability by the inclusion of B-type natriuretic peptide level. Am Heart J 2016;171:33-9.

12 Komatsu T, Onda T, Murayama G, et al. Predicting bacteremia based on nurse-assessed food consumption at the time of blood culture. $J$ Hosp Med 2012;7:702-5.

13 Kawasaki Y, Kojima Y, Akamatsu R. Measuring patient dietary intake using visual estimation methods in Japanese hospitals. Top Clin Nutr 2016;31:335-45.

14 Malone A, Hamilton C. The Academy of nutrition and Dietetics/the American Society for parenteral and enteral nutrition consensus malnutrition characteristics: application in practice. Nutr Clin Pract 2013;28:639-50.

15 Mudge AM, Ross LJ, Young AM, et al. Helping understand nutritional gaps in the elderly (hunger): a prospective study of patient factors associated with inadequate nutritional intake in older medical inpatients. Clin Nutr 2011;30:320-5.

16 Rockwood K, Song X, MacKnight C, et al. A global clinical measure of fitness and frailty in elderly people. CMAJ 2005;173:489-95.

17 Levy WC, Mozaffarian D, Linker DT, et al. The Seattle heart failure model: prediction of survival in heart failure. Circulation 2006;113:1424-33.

18 Shiraishi Y, Kohsaka S, Nagai T, et al. Validation and recalibration of Seattle heart failure model in Japanese acute heart failure patients. $J$ Card Fail 2019;25:1-7

19 Eckardt K-U, Berns JS, Rocco MV, et al. Definition and classification of CKD: the debate should be about patient prognosis--a position statement from KDOQI and KDIGO. Am J Kidney Dis 2009;53:915-20.
20 Tangvik RJ, Tell GS, Eisman JA, et al. The nutritional strategy: four questions predict morbidity, mortality and health care costs. Clin Nutr 2014;33:634-41.

21 Thibault R, Makhlouf A-M, Kossovsky MP, et al. HealthcareAssociated infections are associated with insufficient dietary intake: an observational cross-sectional study. PLoS One 2015;10:e0123695-13.

22 Villet S, Chiolero RL, Bollmann MD, et al. Negative impact of hypocaloric feeding and energy balance on clinical outcome in ICU patients. Clin Nutr 2005;24:502-9.

23 Paulus WJ, Tschöpe C. A novel paradigm for heart failure with preserved ejection fraction: comorbidities drive myocardial dysfunction and remodeling through coronary microvascular endothelial inflammation. J Am Coll Cardiol 2013;62:263-71.

24 Lam CSP, Voors AA, de Boer RA, et al. Heart failure with preserved ejection fraction: from mechanisms to therapies. Eur Heart $J$ 2018;39:2780-92.

25 Don BR, Kaysen G. Serum albumin: relationship to inflammation and nutrition. Semin Dial 2004;17:432-7.

26 Wren AM, Seal LJ, Cohen MA, et al. Ghrelin enhances appetite and increases food intake in humans. J Clin Endocrinol Metab 2001;86:5992-5.

27 Chen Y, Ji X-wu, Zhang A-yuan, et al. Prognostic value of plasma ghrelin in predicting the outcome of patients with chronic heart failure. Arch Med Res 2014;45:263-9.

28 Tsuchihashi M, Tsutsui $\mathrm{H}$, Kodama K, et al. Clinical characteristics and prognosis of hospitalized patients with congestive heart failure. Jpn Circ J 2000;64:953-9.

29 Fried LP, Tangen CM, Walston J, et al. Frailty in older adults: evidence for a phenotype. J Gerontol A Biol Sci Med Sci 2001:56:M146-57.

30 Fried LP, Ferrucci L, Darer J, et al. Untangling the concepts of disability, frailty, and comorbidity: implications for improved targeting and care. J Gerontol A Biol Sci Med Sci 2004;59:M255-63.

31 Tanaka S, Kamiya K, Hamazaki N, et al. Incremental value of objective frailty assessment to predict mortality in elderly patients hospitalized for heart failure. J Card Fail 2018;24:723-32.

32 Vitale C, Spoletini I, Rosano GM. Frailty in heart failure: implications for management. Card Fail Rev 2018;4:104-6.

33 Jabbour A, Chang S, Wilhelm K, et al. The prevalence and prognostic significance of frailty in patients with advanced heart failure referred for heart transplantation. Transplantation 2015;100:429-36.

34 Douglas CC, Lawrence JC, Bush NC, et al. Ability of the Harris Benedict formula to predict energy requirements differs with weight history and ethnicity. Nutr Res 2007;27:194-9.

35 Maisel A, Xue Y, Greene SJ, et al. The potential role of natriuretic peptide-guided management for patients hospitalized for heart failure. J Card Fail 2015;21:233-9.

36 Francis GS, Felker GM, Tang WHW. A test in context: critical evaluation of natriuretic peptide testing in heart failure. $J \mathrm{Am}$ Coll Cardiol 2016;67:330-7. 